\title{
High-Directivity Antenna Array Based on Artificial Electromagnetic Metamaterials with Low Refractive Index
}

\author{
Zhigang Xiao, Jiang Yao, and Shaoquan Yin \\ Physics and Electron Communication Department, Leshan Normal University, Leshan, Sichuan 614004, China \\ Correspondence should be addressed to Zhigang Xiao; zhigangxiaols@gmail.com
}

Received 25 March 2015; Accepted 28 May 2015

Academic Editor: Weiren Zhu

Copyright (C) 2015 Zhigang Xiao et al. This is an open access article distributed under the Creative Commons Attribution License, which permits unrestricted use, distribution, and reproduction in any medium, provided the original work is properly cited.

\begin{abstract}
Planar metamaterials (MTMs) with low refractive index are proposed as a cover in a high-gain patch antenna array configuration. This MTMs array antenna has the following features: the number of array elements significantly decreases compared with the conventional array; the elements spacing is larger than a wave length by far; the feeding network is simpler. MTMs are made of two layers of periodic square metallic grids and placed above the feeding array. With the same aperture size, the directivity of MTMscover antenna array is higher than the conventional antenna array. The simulation results show that an array of $2 \times 2$ patch elements integrated with MTMs yields about $26 \mathrm{~dB}$ of directivity which is higher than that of conventional $8 \times 8$ patch array. Furthermore, on the condition of the same aperture size, an array patch with $4 \times 4$ elements integrated with the MTMs-cover has an equivalent gain compared with the conventional patch array with $16 \times 16$ array elements. Obviously, the former has a simpler feeding network and higher aperture efficiency. The experimental work has verified that the $2 \times 2$ array case and the measured results have good agreement with the simulation.
\end{abstract}

\section{Introduction}

The traditional antenna technology to achieve the high directivity needs multiple radiating elements to fabricate the antenna array. The spacing between radiating elements is normally $50 \%$ to $70 \%$ of a wavelength. If the number of the array elements increases, it will increase propagation loss and make the feeding network become more complicated [1]. Emergence of the metamaterials (MTMs) provides a new technology to improve antenna innovation. A large number of research works have been achieved in obtaining the high directivity of antenna by using MTMs [2-9]. MTMs can be fabricated through various ways such as photonic crystal $[2,3]$, electromagnetic band gap (EBG) structure, frequency selective surface (FSS) [4-6], and other periodic artificial material which can be designed to have a low refractive index [7-9].

An ordinary microstrip patch antenna has the gain of about $7 \mathrm{~dB}$. If a single patch is covered by a layer of MTMs, the gain will increase to about $20 \mathrm{~dB}$. Compared with the conventional antenna array, a single feed source integrated with the MTMs-cover can obtain the same antenna gain or higher. However, the antenna with MTMs-cover has higher aperture efficiency and simpler structure. And the manufacturing is relatively easy. If the feeding source is antenna array with MTMs-cover, the array becomes a sparse array of which the elements' spacing is more than one wavelength. The antenna array to meet the above conditions can achieve higher antenna gain. Meanwhile, because the number of the elements decreases, the array elements spacing becomes larger which results in the feeding network becoming simpler.

This paper presents a high-directivity antenna array model based on MTMs with low refractive index. A fourelement array $(2 \times 2$ patch) excited by the coaxial probe is used as the source, and MTMs, which are made of the planar periodic metallic grids and have a low refractive index, are used as the cover. The simulated and measured results show that, on the condition of the same aperture size, the $2 \times$ 2 microstrip patches array integrated with MTMs-covers has high directivity about $26 \mathrm{~dB}$ which is better than the conventional antenna array. The $4 \times 4$ patch array with MTMs-cover may replace the conventional $16 \times 16$ patch array, and they have similar high directivity. 


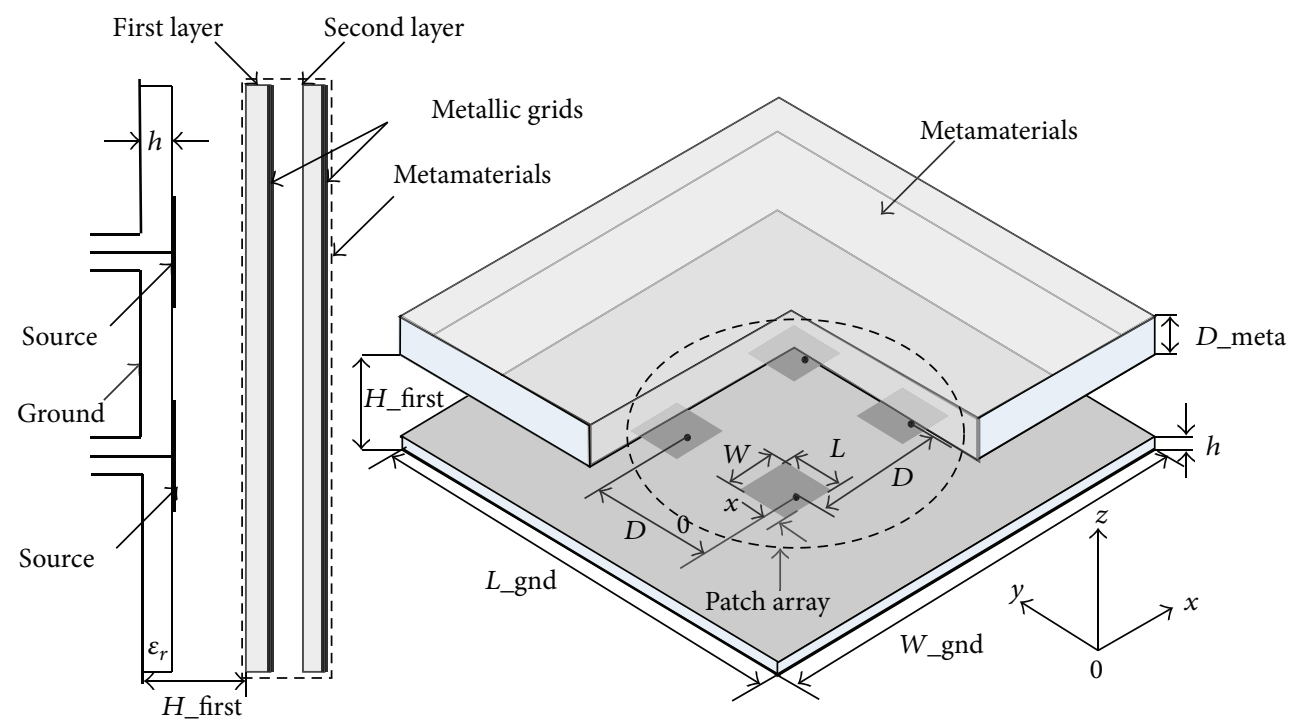

FIGURE 1: $2 \times 2$ element antenna array integrated with MTMs-cover.

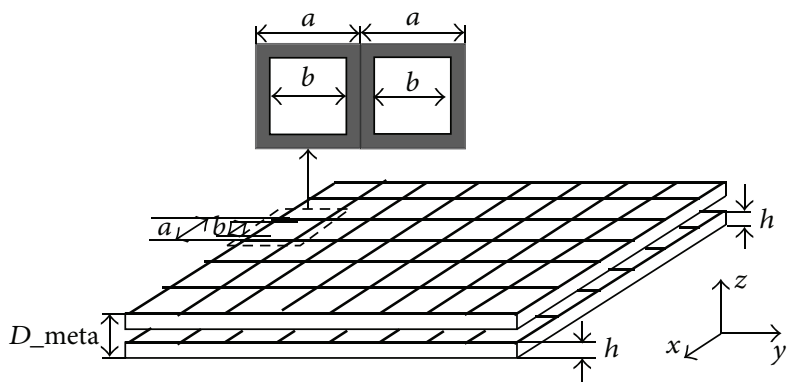

FIgure 2: Metallic grids structure with low refractive index.

\section{Antenna Array Structure and Simulation Results}

The $2 \times 2$ patch array integrated with planar MTMs-cover is shown in Figure 1 . The source is the $2 \times 2$ element patch array and fed by the coaxial probe, respectively. MTMs-cover with low refractive index is made of two layers of planar periodic metallic grids, as shown in Figure 2. The parameters of structure are designed carefully to operate at the Ku band and $14.6 \mathrm{GHz}$ is suitable. The patch arrays and metallic grids are printed on a $1.5 \mathrm{~mm}$ thick microwave printed circuit board (PCB) substrate $\left(\varepsilon_{r}=2.2\right)$. The metallic grid cell size is shown as follows: $a=5.8 \mathrm{~mm}$ and $b=4.6 \mathrm{~mm}$. The thickness of MTMs-cover is D_meta $=5.4 \mathrm{~mm}$, and the height from MTMs-cover to the ground is $H_{-}$first $=7.1 \mathrm{~mm}$. The operating frequency of the array is $14.6 \mathrm{GHz}$. The patch elements have the same parameters: $(L, W, x 0)=(5.2 \mathrm{~mm}, 6.1 \mathrm{~mm}, 1.8 \mathrm{~mm})$, and element spacing is $D=2.5 \lambda_{(14.6 \mathrm{GHz}\}}$. The ground has the size $\left(L_{-}\right.$gnd, $\left.W_{\text {-gnd }}\right)=(240 \mathrm{~mm}, 240 \mathrm{~mm})$, which is slightly larger than the area $(230 \mathrm{~mm} \times 230 \mathrm{~mm})$ size of forty cells of the metallic grids.

Array element spacing $(D)$ is a very important parameter. For the conventional antenna arrays, element spacing is about
$0.5 \lambda \sim 1.0 \lambda$, where $\lambda$ is the operating wavelength. If the spacing is larger than this range, the grating lobe is obvious. If the spacing is lower than this range, the mutual coupling becomes strong. Therefore, the element spacing is defined at approximately $0.7 \lambda$. However, for the antenna array with MTMs-cover, the spacing is considerably larger than $1.0 \lambda$. Our research results show that the antenna array with MTMscover can show its value only when the element spacing is about $D=2.5 \lambda_{(14.6 \mathrm{GHz}\}}$.

The radiation performance of the conventional $2 \times 2$ element patch array and the $2 \times 2$ element array integrated with MTMs-cover (both having the same element spacing $\left.D=2.5 \lambda_{(14.6 \mathrm{GHz}\}}\right)$ is analyzed by CST Microwave Studio. (Here, authors would like to express their sincere gratitude to the CST Company.) Figure 3 shows the comparison of their radiation patterns. The red curve indicates the case of conventional patch array. Without MTMs-cover, if the element spacing is greatly larger than $2.0 \lambda$, the grating lobes and the directivity performance become worse, respectively. However, the array with MTM-cover has good directivity and lower side lobes.

Figure 4 shows the three-dimensional radiation patterns of the two antenna arrays. It is obvious that MTMs-cover has 


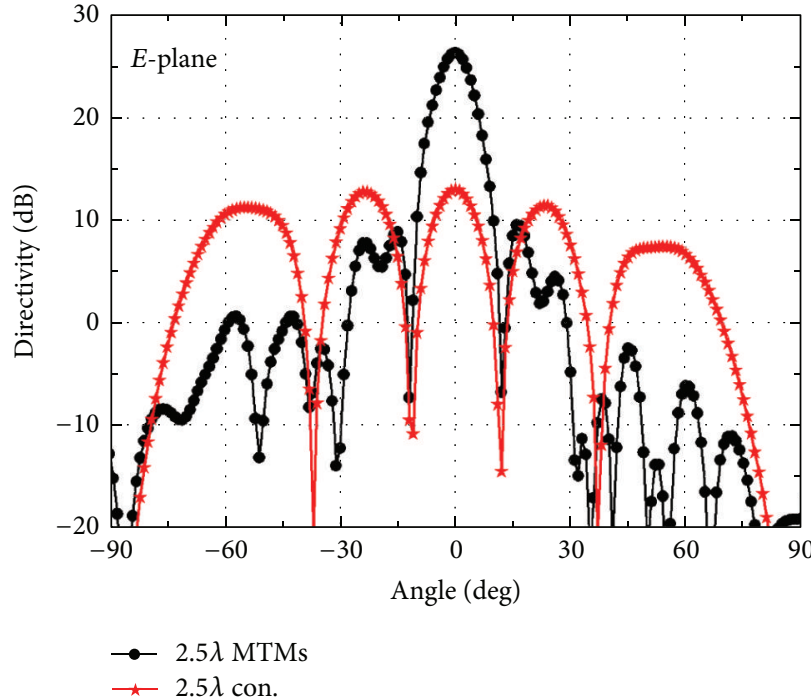

(a) E-plane

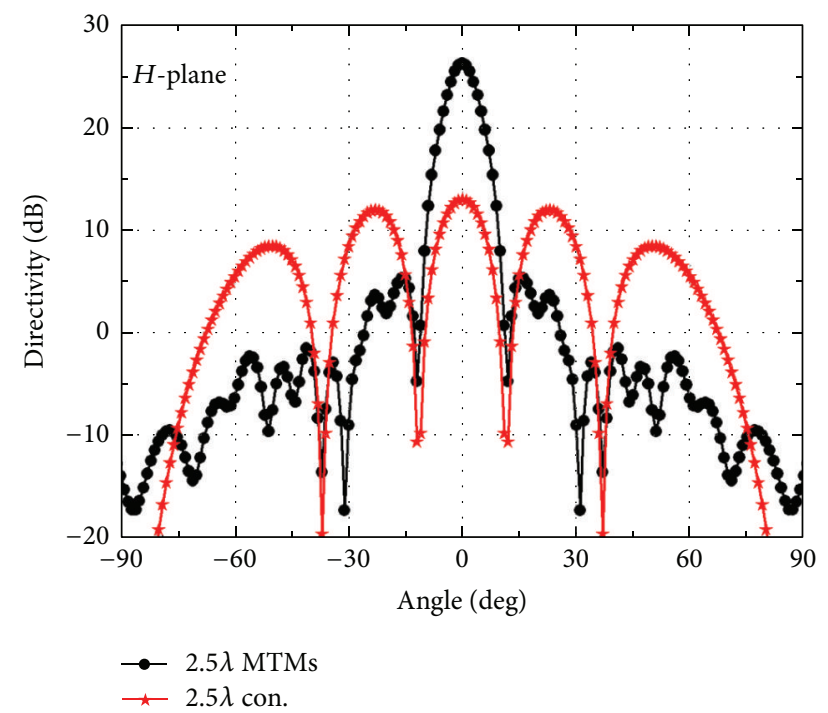

(b) H-plane

FIGURE 3: Radiation patterns of MTMs-cover array and the conventional type $\left(D=2.5 \lambda_{(14.6 \mathrm{GHz}\}}\right)$ : (a) $E$-plane; (b) $H$-plane.

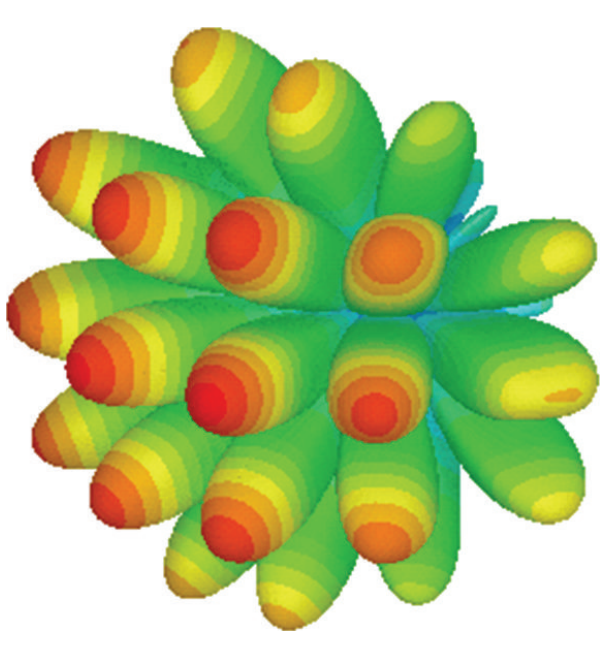

(a) Conventional four-element antenna array
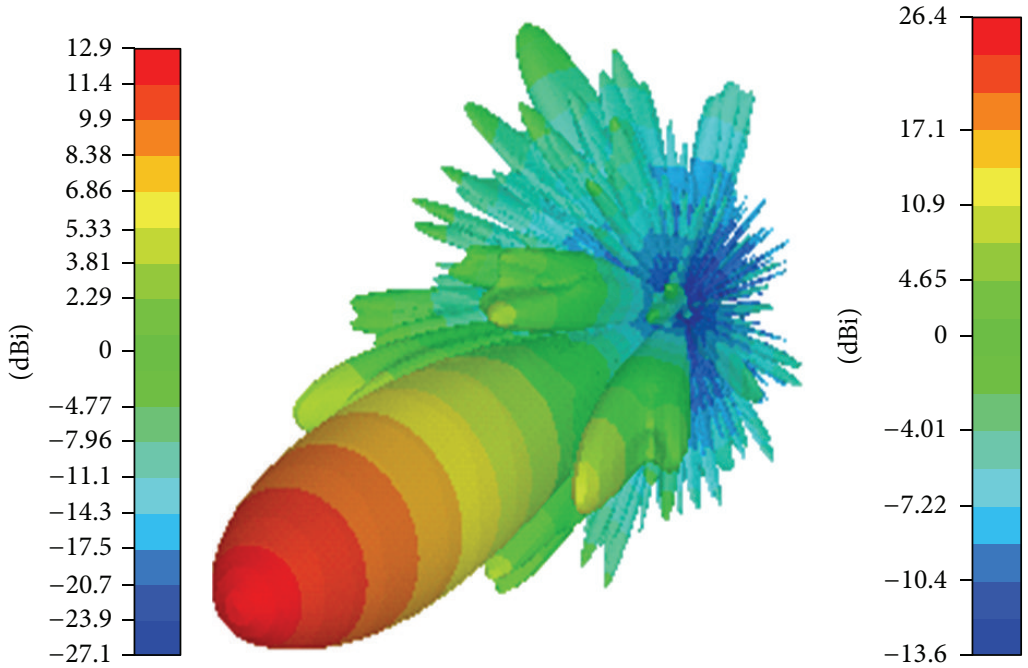

(b) Four-element antenna array integrated with MTMs-cover

FIgURE 4: Three-dimensional radiation patterns of MTMs-cover array and the conventional type: (a) conventional $2 \times 2$ element antenna array; (b) $2 \times 2$ element antenna array with MTMs-cover.

effect similar to "optical lens," which is capable of focusing the diffuse scattering electromagnetic wave for directive radiation.

Figure 5 illustrates the return loss of conventional patch and MTMs-cover type. The central operating frequency is $14.6 \mathrm{GHz}$. The conventional array has wider bandwidth. Because of the existence of MTMs-cover, the antenna impedance matching becomes worse when operating frequency is lower than the $14.4 \mathrm{GHz}$. Antenna impedance mismatching can be improved by adjusting parameters of the metallic grids.

The directivity of antenna array is calculated as follows: $D_{\text {dir }}(\mathrm{dB})=10 \log \left(k \cdot 4 \pi A / \lambda_{0}^{2}\right)$, where $A$ is the physical area of the antenna ground and $k(0 \sim 1)$ is the effective coefficient. Given $k=1$, the theoretical value of the maximum directivity is $D_{\max }(\mathrm{dB})=10 \log \left(4 \pi A / \lambda_{0}^{2}\right)$. Here the area of the aperture is $A=240 \mathrm{~mm} \times 240 \mathrm{~mm}$ and $\lambda_{0}=20.55 \mathrm{~mm}(14.6 \mathrm{GHz})$; therefore the maximum directivity is $D_{\max }(\mathrm{dB})=32.3 \mathrm{~dB}$. The simulation result is $D_{\text {dir }}(\mathrm{dB})=26.3 \mathrm{~dB}$. The aperture efficiency of the antenna is $k=\log ^{-1}\left(D_{\text {dir }} / 10\right) / \log ^{-1}\left(D_{\max } / 10\right)=$ 0.25 . The aperture efficiency is relatively lower. The result shows that the aperture area in this case is larger for the $2 \times$ 2 array. If the higher directivity is not necessary, the physical area of the antenna can be properly reduced to increase the aperture efficiency. On the other hand, to keep the same physical area of the antenna and increase the number of 


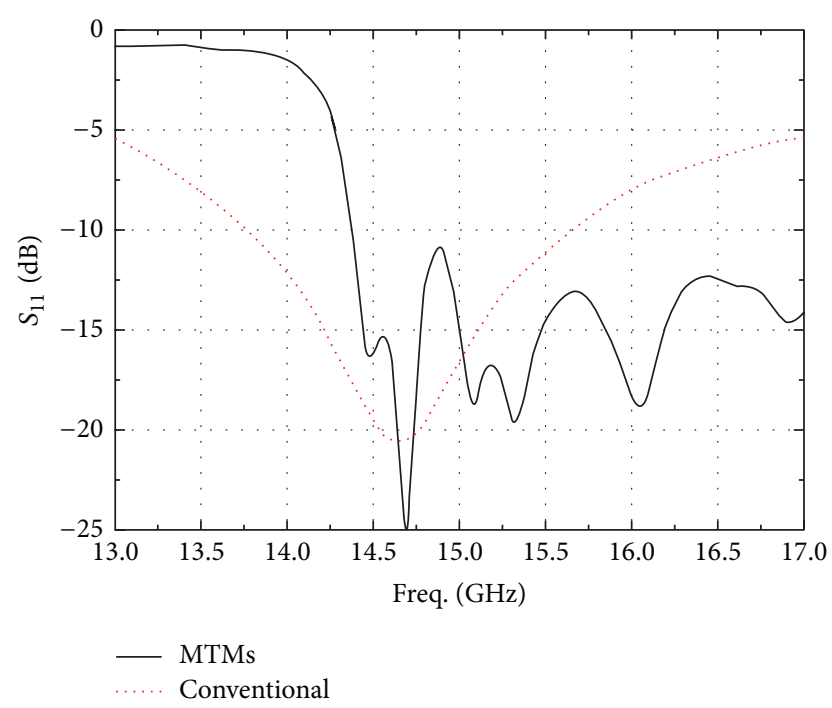

FIGURE 5: Return loss comparison of the conventional patch and MTM-cover type.

the array elements, it can be ensured that the antenna has higher directivity with improved aperture efficiency.

\section{Experimental Results}

In order to verify the theoretical analysis results, the antenna prototype is fabricated according to the structure parameters of the model. Figure 6 shows the $2 \times 2$ element patch source, the assembled antenna with MTMs-cover, and the antenna in the anechoic chamber. The $2 \times 2$ element patch array is fed by the $50 \mathrm{ohm}$ coaxial line, and the feeding signal is provided through a power divider. Two layers of the metallic grids are fixed by using twelve nylon rods and installed to the ground plate.

Figure 7 shows the radiation patterns of the antenna prototype. Simulation results are in good agreement with measurements, which shows that the antenna design obtains success. It is worth noting that the fabrication error of prototype with two-layer metallic grids will affect the test results. In order to achieve good results, the prototype assembly need to be finely adjusted, which avoids as much as possible the physical influence.

\section{Discussion}

Through increasing the number of array elements, the conventional antenna array can obtain better directivity. Similarly, the method is also applicable to the antenna array with MTMs-cover. In $2 \times 2$ case, if the aperture area of the antenna with MTMs-cover remains invariable and the number of array elements increases to $4 \times 4$, the antenna aperture efficiency will be enhanced obviously. The simulated radiation patterns of $2 \times 2$ array and $4 \times 4$ array with MTMs-cover are shown in Figure 8, respectively. The operating frequency is $14.6 \mathrm{GHz}$ and the element spacing is
$D=2.5 \lambda_{(14.6 \mathrm{GHz}\}}$. The $2 \times 2$ array has a directivity of about $24.8 \mathrm{~dB}$, while the directivity of $4 \times 4$ array is improved to approximately $29.14 \mathrm{~dB}$. The aperture efficiency of $4 \times 4$ array is about 0.48 . It is clear that increasing array elements improves the aperture efficiency obviously.

For MTMs-cover antenna array, using more array elements is a better approach to improve the antenna aperture efficiency. In addition, selection of the array element spacing is also a critical factor. Figure 9 shows that the aperture efficiency of $2 \times 2$ elements and $4 \times 4$ elements antenna array varies with the array element spacing change, respectively. The antenna array with MTMs-cover must be a sparse array, and the element spacing is in a reasonable range. In this case, the optimum spacing is about $2.5 \lambda_{14.6 \mathrm{GHz}}$; otherwise it cannot achieve the expected results.

Without considering the transmission loss, when the aperture area is $240 \mathrm{~mm} \times 240 \mathrm{~mm}$, the maximum theoretical directivity of conventional $16 \times 16$ element antenna array is approximately $32.3 \mathrm{~dB}$. Considering all the loss, the antenna array aperture efficiency is about $-3 \mathrm{~dB}$, so the directivity is about $29.3 \mathrm{~dB}$, which is equivalent to that of $4 \times 4$ elements antenna array with MTMs-cover. However, the $4 \times 4$ elements antenna array with MTMs-cover has simpler and easier fabricated feeding network compared with conventional array. These advantages show that the $4 \times 4$ elements antenna array with the simper feeding network can replace the conventional $16 \times 16$ element antenna array, so as to achieve the goal of making complex design become simpler.

\section{Conclusion}

The patch antenna array integrated with MTMs-cover proposed in this paper can markedly improve the antenna aperture efficiency and the antenna directivity. This type of antenna has the following characteristics: reduction of the number of array elements does not reduce the antenna directivity; increasing of the spacing of array element is beneficial to simplify a feeding network so as to reduce cost or provide extra space to other components on PCB substrate. A conventional $16 \times 16$ element antenna array can be replaced by a $4 \times 4$ elements antenna with MTMs-cover, and they have the same high directivity. The research results have a potential application to a large printed high-gain antenna array system.

\section{Conflict of Interests}

The authors declare that there is no conflict of interests regarding the publication of this paper.

\section{Acknowledgments}

This work was supported by Major Project of Education Department in Sichuan Foundation (no. 11ZA157). In addition, the authors would like to express their sincere gratitude 


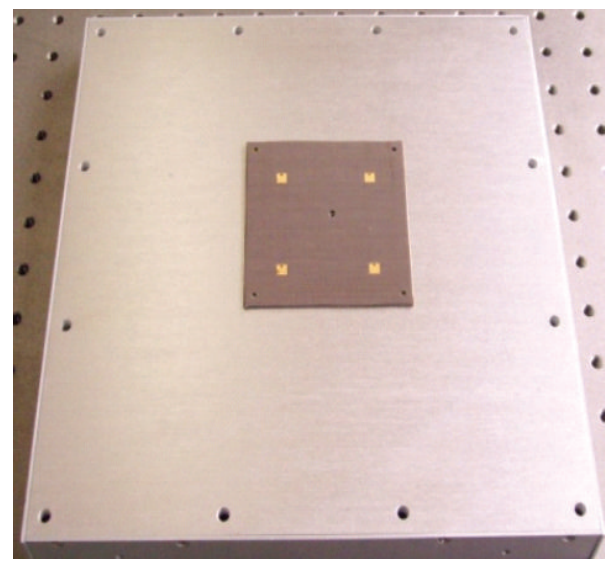

(a)

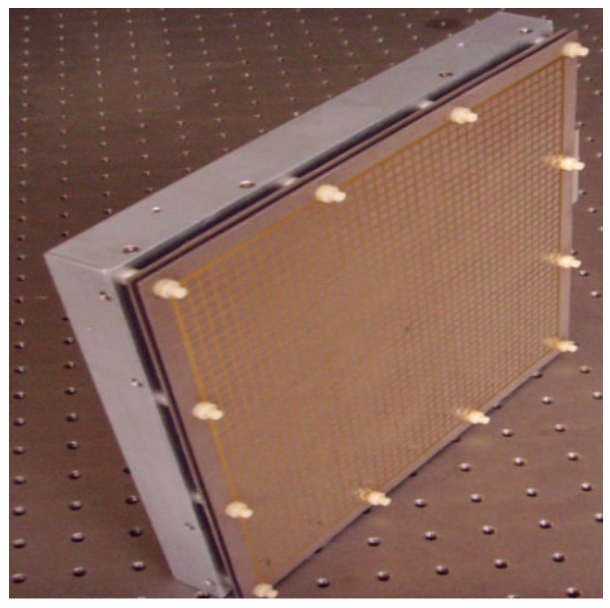

(c)

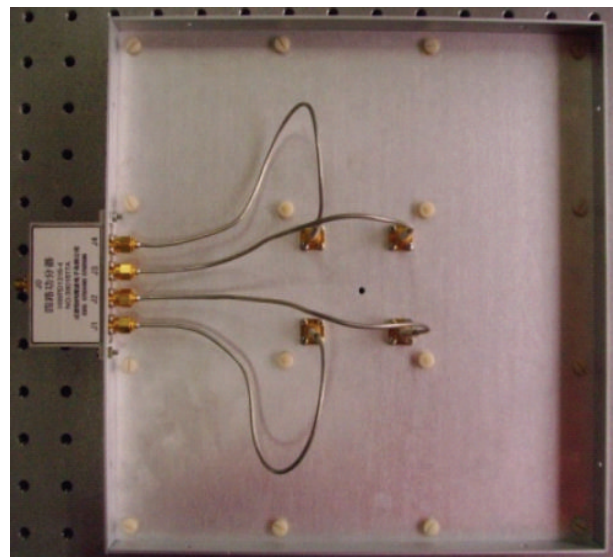

(b)

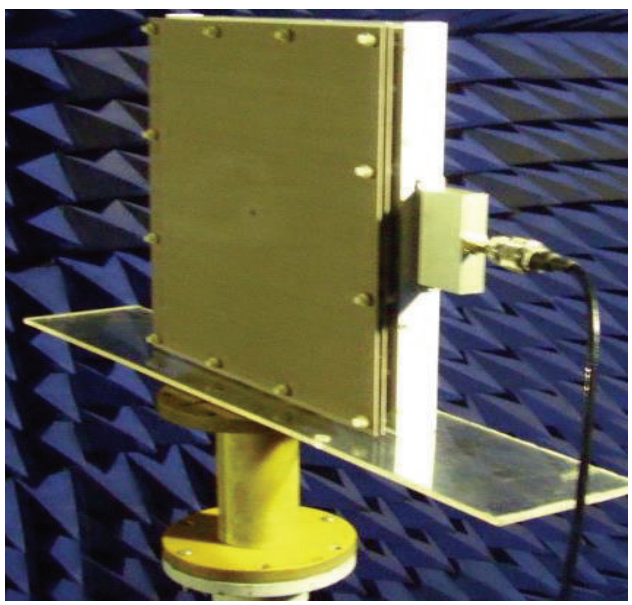

(d)

FIGURE 6: Photographs of patch antenna array and measured status in the anechoic chamber: (a) $2 \times 2$ element patch antenna array; (b) back view of the antenna array; (c) patch array with MTMs-cover; (d) MTMs-cover antenna in the anechoic chamber.

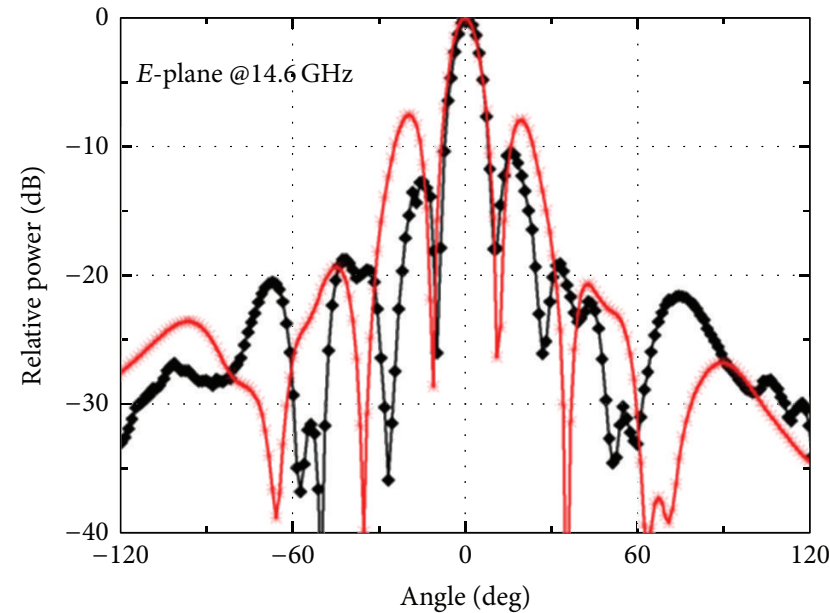

$\rightarrow$ Measured

* Simulated

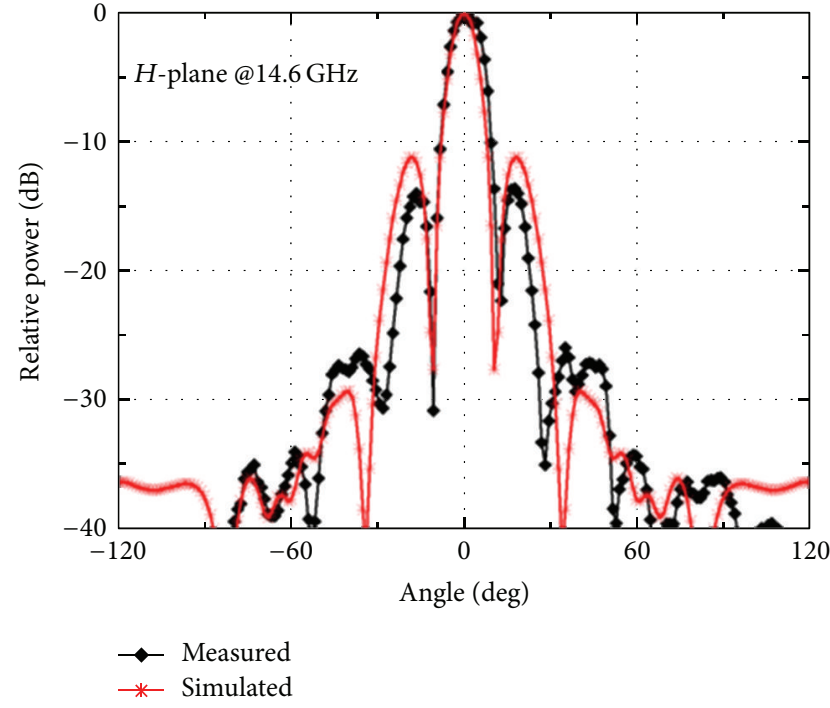

(b)

FIgURE 7: Radiation patterns of $2 \times 2$ element patch array with MTMs-cover: (a) $E$-plane; (b) $H$-plane. 


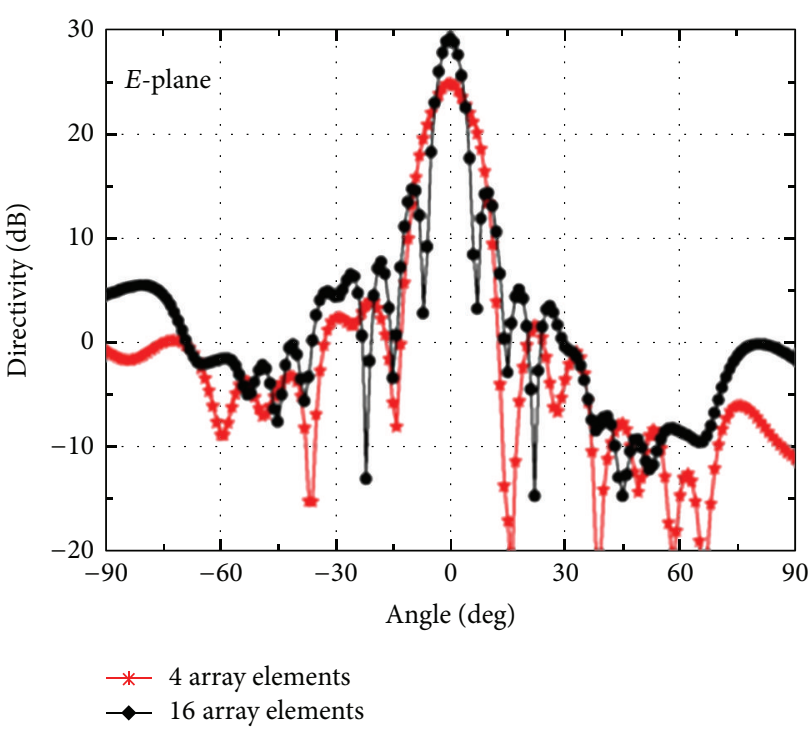

(a)

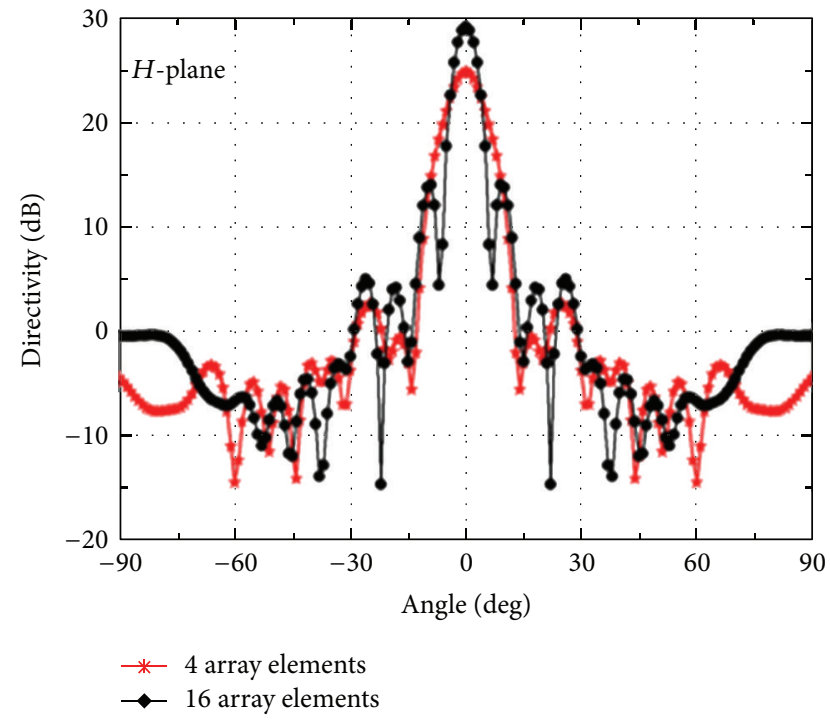

(b)

FIGURE 8: Antenna radiation patterns of the two arrays with MTMs-cover $\left(D=2.0 \lambda_{14.6 \mathrm{GHz}}\right)$ : (a) $E$-plane; (b) $H$-plane.

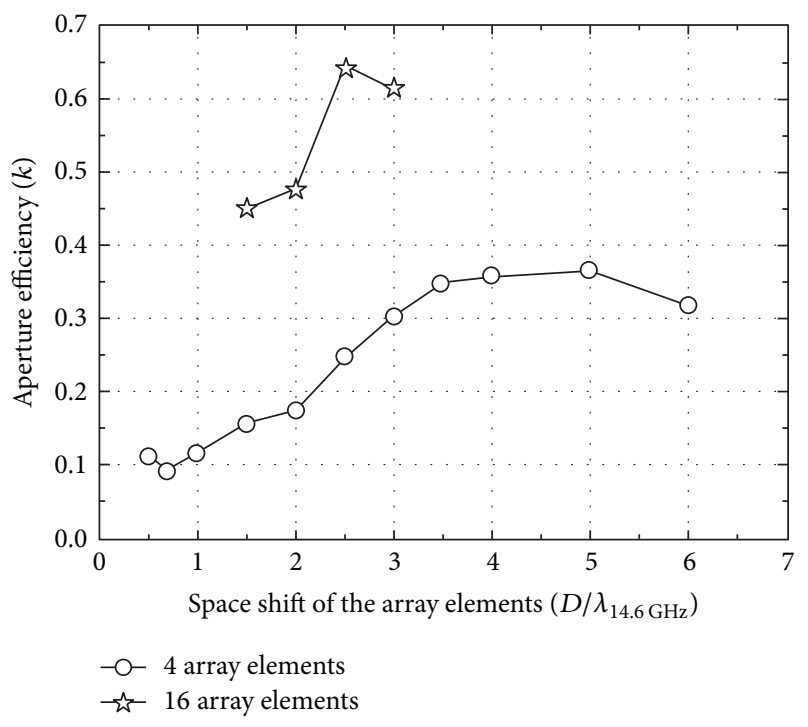

FIGURE 9: Aperture efficiency and element spacing of the two MTMs-cover arrays.

to the CST China, for providing the evaluated software for the simulation.

\section{References}

[1] E. Levine, G. Malamud, S. Shtrikman, and D. Treves, "A study of microstrip array antennas with the feed network," IEEE Transactions on Antennas and Propagation, vol. 37, no. 4, pp. 426-434, 1989.

[2] M. Thèvenot, M. S. Denis, A. Reineix, and B. Jecko, "Design of a new photonic cover to increase antenna directivity," Microwave and Optical Technology Letters, vol. 22, no. 2, pp. 136-139, 1999.
[3] B. Temelkuran, M. Bayindir, E. Ozbay et al., "Photonic crystalbased resonant antenna with a very high directivity," Journal of Applied Physics, vol. 87, no. 1, pp. 603-605, 2000.

[4] A. R. Weily, L. Horvath, K. P. Esselle, B. C. Sanders, and T. S. Bird, "A planar resonator antenna based on a woodpile EBG material," IEEE Transactions on Antennas and Propagation, vol. 53, no. 1, pp. 216-223, 2005.

[5] S. Wang, A. P. Feresidis, G. Goussetis, and J. C. Vardaxoglou, "High-gain subwavelength resonant cavity antennas based on metamaterial ground planes," IEE Proceedings: Microwaves, Antennas and Propagation, vol. 153, no. 1, pp. 1-6, 2006.

[6] Y. J. Lee, J. Yeo, R. Mittra, and W. S. Park, "Design of a high-directivity Electromagnetic Band Gap (EBG) resonator antenna using a frequency-selective surface (FSS) superstrate," Microwave and Optical Technology Letters, vol. 43, no. 6, pp. 462-467, 2004.

[7] S. Enoch, G. Tayeb, P. Sabouroux, N. Guérin, and P. Vincent, "A metamaterial for directive emission," Physical Review Letters, vol. 89, no. 21, pp. 213902-1-213902-4, 2002.

[8] Q. Wu, P. Pan, F.-Y. Meng, L.-W. Li, and J. Wu, "A novel flat lens horn antenna designed based on zero refraction principle of metamaterials," Applied Physics A: Materials Science and Processing, vol. 87, no. 2, pp. 151-156, 2007.

[9] H. Xu, Z. Zhao, Y. Lv, C. Du, and X. Luo, "Metamaterial superstrate and electromagnetic band-gap substrate for high directive antenna," International Journal of Infrared and Millimeter Waves, vol. 29, no. 5, pp. 493-498, 2008. 

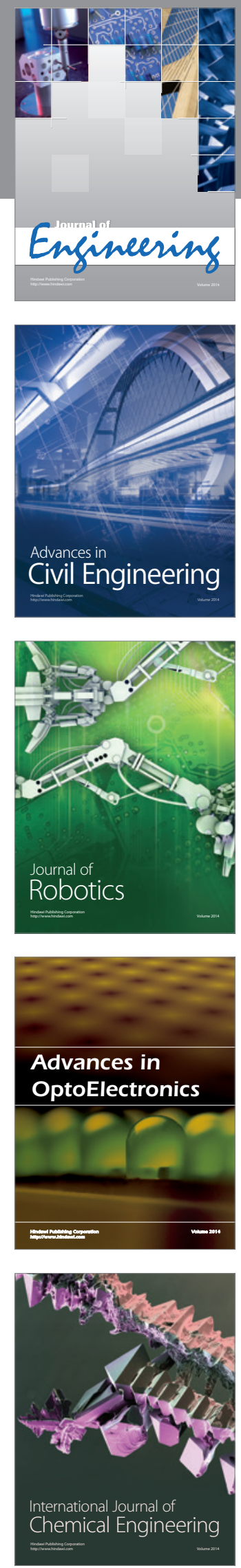

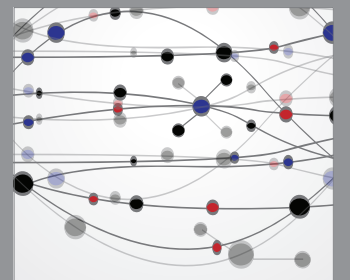

The Scientific World Journal
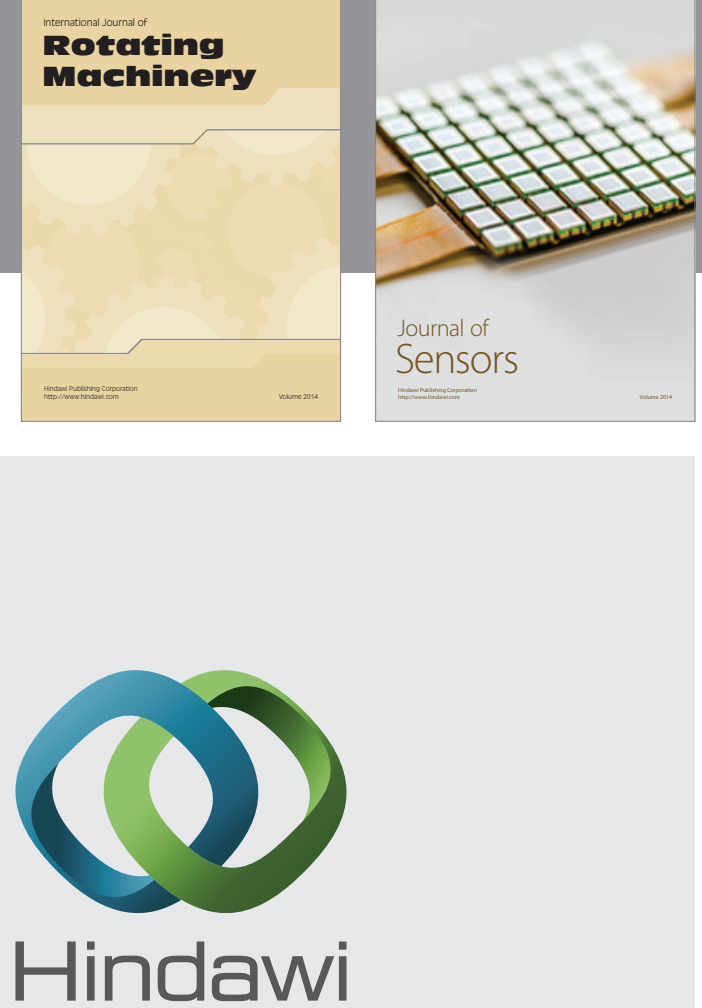

Submit your manuscripts at http://www.hindawi.com
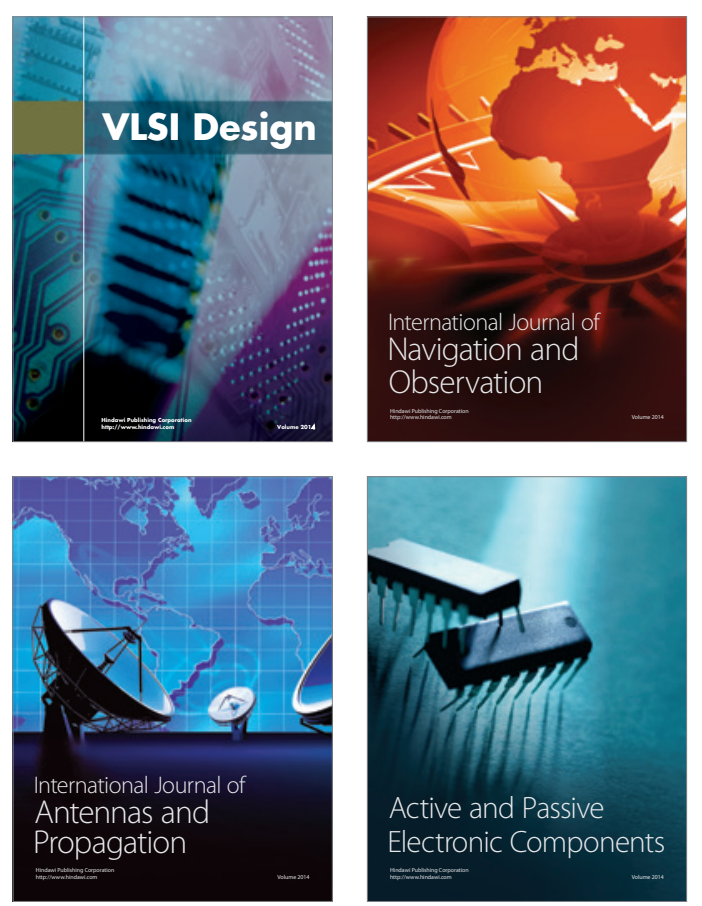
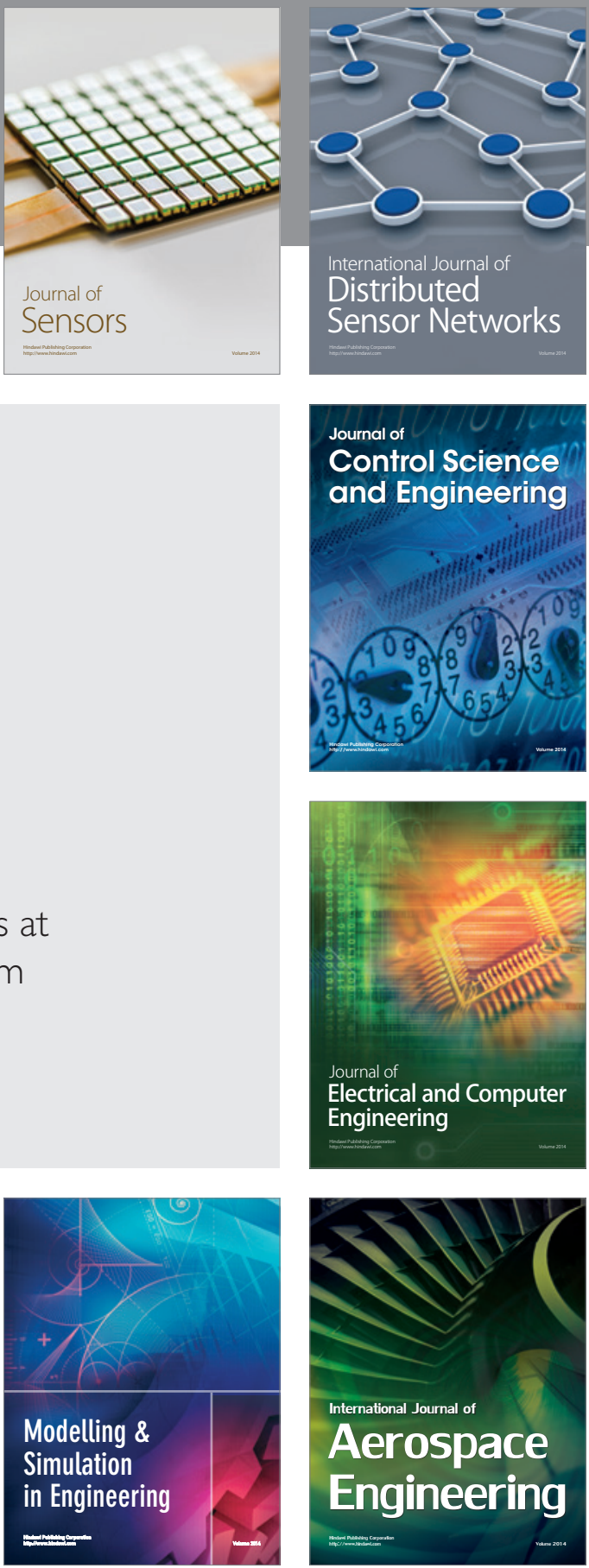

Journal of

Control Science

and Engineering
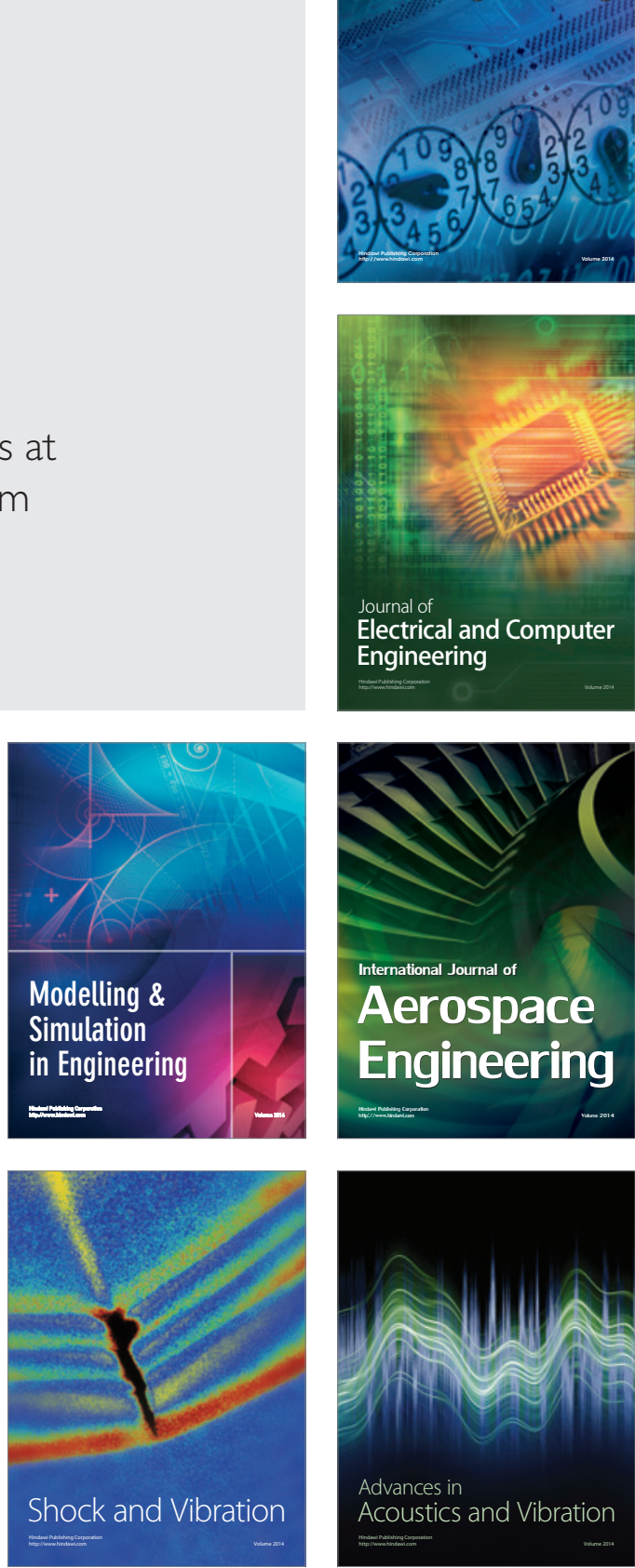